\title{
Úlcera gastroyeyunal perforada en paciente con derivación gastroyeyunal posterior a abdominoplastia en posquirúrgico inmediato. Un abordaje preservando lo estético
}

\author{
Perforated marginal ulcer in a patient with gastro-yeyunal diversion after \\ abdominoplasty in immediate post-surgery. An approach preserving the aesthetic \\ Juan Antonio López Corvalá, ${ }^{*}$ Fernando Guzmán Cordero, ${ }^{\ddagger}$ Jesús Arturo Medina Ramírez, ${ }^{\S}$ \\ Eduardo Aguilar Díaz, ${ }^{\S}$ Sergio Cabral Elizondo§ \\ * Jefe del Departamento de Cirugía de Mínima Invasión y Cirugía Bariátrica. \\ ‡ Profesor del curso de Cirugía de Mínima Invasión y Cirugía Bariátrica. \\ $\S$ Cirujano General en adiestramiento en Cirugía de Mínima Invasión y Cirugía Bariátrica.
}

Hospital Ángeles Tijuana, Baja California.

RESUMEN

Introducción: La úlcera marginal posterior a derivación gastroyeyunal se reporta en $6 \%$ de los pacientes, de los cuales se perfora el $1 \%$. El abordaje quirúrgico de elección en estos casos es el laparoscópico. Reoperar a un sujeto de manera urgente con abordaje laparoscópico en el posquirúrgico inmediato de cirugía estética, por ejemplo con abdominoplastia con plicatura de músculos rectos, implica un gran reto para el diagnóstico oportuno y el abordaje. Una de las complicaciones de la derivación gastroyeyunal (DGY) en cirugía bariátrica es la úlcera marginal perforada (UMP), la cual lleva al paciente a cirugía de urgencia. Presentamos un reporte de caso de esta complicación en su posquirúrgico inmediato de abdominoplastia. Se decide tratamiento quirúrgico con abordaje laparoscópico no convencional desmantelando abdominoplastia y colocando puertos de trabajo directamente sobre la aponeurosis. Conclusión: Para pacientes con derivación gastroyeyunal y úlcera marginal perforada, recomendamos en posquirúrgico inmediato de abdominoplastia el desmantelamiento del colgajo dermograso y retiro
ABSTRACT

Introduction: MU ulcer after GBYR is reported in $6 \%$ of patients, of which $1 \%$ are perforated. The surgical approach of choice in these patients is laparoscopic. Reoperating a patient urgently with a laparoscopic approach in the immediate postoperative period of cosmetic surgery, such as tummy tuck abdominoplasty, involves a great challenge for both timely diagnosis and approach. One of the complications of the Roux-en-Y gastric bypass surgery (GBYR) in bariatric surgery is the marginal ulcer $(M U)$, being the perforation that leads the patient to emergency surgery. We present a case report of perforated marginal ulcer (PMU) in a patient with a history of GBYR 2 years ago, in his immediate post-surgical. Surgical treatment with an unconventional laparoscopic approach is decided by dismantling a tummy tuck and placing work ports directly on the aponeurosis. Conclusion: We recommend in a patient with GBYR and MPU in immediate post-surgical abdominoplasty the dismantling of the dermogreasy flap and removal of rectus plication to perform the laparoscopic approach directly on the aponeurosis.

\section{WWVW.medigraphic.orgnim}

Recibido: 26/01/2020. Aceptado: 28/09/2020.

Correspondencia: Dr. Juan Antonio López Corvalá. E-mail: drcorvala@grupolap.com Dr. Jesús Arturo Medina Ramírez. E-mail: drjesusmedinaramirez@hotmail.com

Citar como: López CJA, Guzmán CF, Medina RJA, Aguilar DE, Cabral ES. Úlcera gastroyeyunal perforada en paciente con derivación gastroyeyunal posterior a abdominoplastia en posquirúrgico inmediato. Un abordaje preservando lo estético. Rev Mex Cir Endoscop. 2020; 21 (4): 217-220. https://dx.doi.org/10.35366/101223 
de plicatura de rectos para realizar el abordaje laparoscópico directamente sobre la aponeurosis.

Palabras clave: Abdominoplastia, mínima invasión, derivación gastroyeyunal, úlcera.
Keywords: Tummy tuck, minimally invasive, gastrojejunal bypass, ulcer.
Abreviaturas:

DGY = Derivación gastroyeyunal.

$\mathrm{UM}=$ Úlcera marginal.

$\mathrm{UMP}=$ Úlcera marginal perforada.

\section{INTRODUCCIÓN}

Existen varios procedimientos para el tratamiento de la obesidad mórbida, entre los que se encuentra la derivación gastroyeyunal (DGY), la cual puede presentar como complicación la úlcera marginal (UM). ${ }^{1}$

La UM se desarrolla en la anastomosis gastroyeyunal con una incidencia que se encuentra entre 0.6 y $6 \%,{ }^{2}$ sus síntomas principales son dolor retro esternal y dolor abdominal; sin embargo, hasta $61 \%$ de los casos se encuentran asintomáticos. ${ }^{2}$ Después de que se diagnostica UM, 17\% de los pacientes tendrán que ser intervenidos quirúrgicamente; las principales indicaciones relacionadas a esto son: perforación, fístula gastro-gástrica, sangrado activo y falta de respuesta al tratamiento médico. ${ }^{2}$

La etiología de la UM es multifactorial, identificando factores de riesgo como el abuso de antinflamatorios no esteroideos (AINE), corticoesteroides, nicotina, reacción a cuerpo extraño como la línea de sutura y Helicobacter pylori. ${ }^{3}$ En algunos casos, la UM amerita manejo quirúrgico debido a persistencia de sintomatología a pesar del tratamiento médico o a complicaciones como la perforación, cuya incidencia es de $0.83 \%$. $^{3}$ De los sujetos con UM que se perforan, $20 \%$ no cuenta con factores predisponentes.

El objetivo de este estudio es presentar un caso poco frecuente, úlcera marginal perforada (UMP) con un abordaje quirúrgico diferente, ya que después de la abdominoplastia con plicatura de rectos en un posoperatorio temprano, el abordaje laparoscópico convencional sería de gran dificultad.

\section{CASO CLÍNICO}

Mujer de 51 años con antecedente de DGY por obesidad mórbida en el año 2012, artroscopía de hombro en el 2014, que presentó como complicación UMP resuelta quirúrgicamente. Su padecimiento actual inicia posterior a abdominoplastia; tres días después se solicita valoración por nuestro servicio debido a cuadro de dolor abdominal intenso de 24 horas de evolución, de predominio en mesogastrio y epigastrio, con irradiación en la totalidad del abdomen.
Cabe mencionar que fue manejada con infusión de ketorolaco $180 \mathrm{mg} 24$ horas después de la abdominoplastia.

A la exploración física, la paciente se encuentra intranquila, con facies dolorosa, abdomen plano, con herida horizontal afrontada de aproximadamente $50 \mathrm{~cm}$ a nivel de pubis, drenaje bilateral colocado debajo de colgajo dermograso con salida de líquido serohemático escaso. Pared abdominal tensa secundaria a abdominoplastia, con signos de irritación peritoneal.

Estudios de laboratorio: Leucocitos de $2.6 / \mu \mathrm{L}$, neutrofilia $79 \%$, tiempo de protrombina 15.2 segundos. Tomografía axial computarizada reporta: aire libre perihepático, líquido libre en cavidad abdominal, niveles hidroaéreos.

Con base en los antecedentes, hallazgos clínicos y estudio de imagen se decide someter a procedimiento quirúrgico de urgencia.

\section{Técnica quirúrgica}

Paciente bajo anestesia total endovenosa en decúbito supino, se desmantela abdominoplastia y se liberan puntos de plicatura de músculos rectos abdominales (Figura 1). Sobre la aponeurosis de la pared abdominal se coloca el primer puerto de $12 \mathrm{~mm}$ (posteriormente mano derecha del cirujano) en línea media supraumbilical para realizar neumoperitoneo con $\mathrm{CO}_{2}$ a 15 mmHg e introducción de óptica para colocar el resto de los puertos (Figura 2); flanco

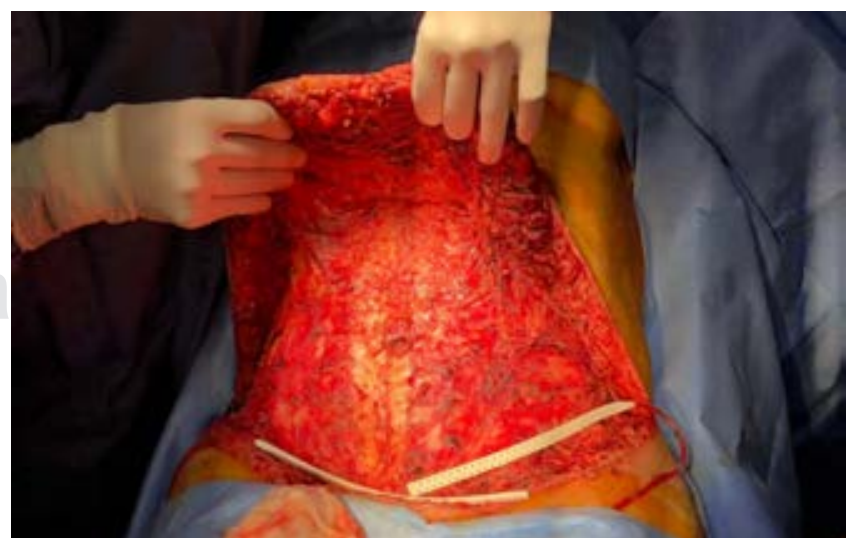

Figura 1: Desmantelamiento de abdominoplastia y exposición de aponeurosis. 


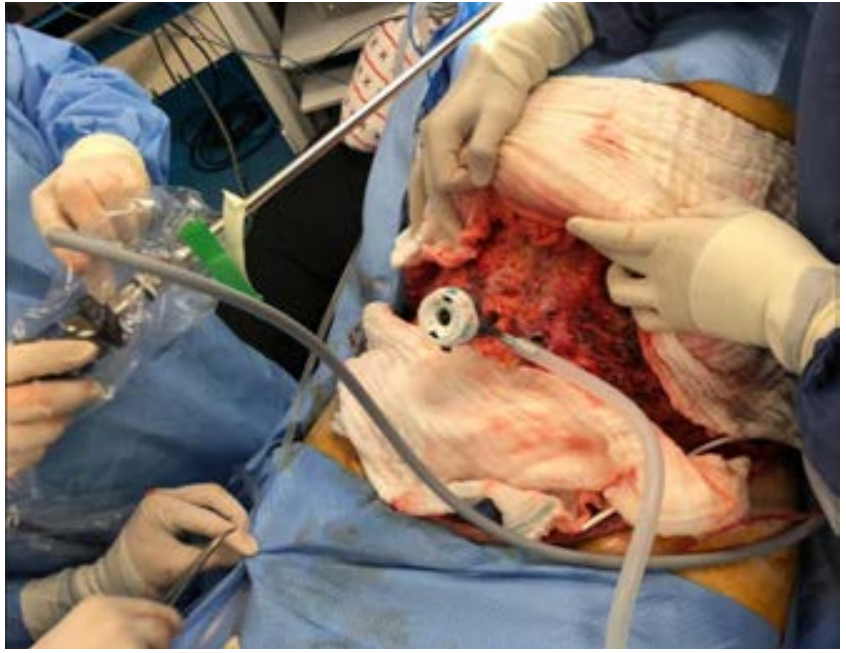

Figura 2: Colocación del primer puerto supraumbilical.

derecho en línea media clavicular $5 \mathrm{~mm}$ (mano izquierda del cirujano), subxifoideo $10 \mathrm{~mm}$ (separador hepático), subcostal izquierdo en línea media clavicular $12 \mathrm{~mm}$ (óptica), y flanco izquierdo $5 \mathrm{~mm}$ (ayudante).

A la exploración, identificamos alrededor de tres litros de líquido inflamatorio purulento distribuidos en la cavidad abdominal, el cual se aspira, así como natas de fibrina y adherencias interasa intestinal. Se identifica reservorio gástrico y se libera utilizando bisturí armónico (Ethicon Endo-Surgery, Cincinnati, OH), se expone perforación a nivel de la anastomosis gastroyeyunal de $1 \mathrm{~cm}$ de diámetro (Figura 3). Se repara con sutura polipropileno 2-0 puntos simples y parche de epiplón. Se realiza prueba de hermeticidad con azul de metileno verificando ausencia de fugas. Por último, lavado con solución fisiológica cinco litros y se coloca drenaje tipo Blake de $19 \mathrm{Fr}$; se cierran los orificios de los puertos con polipropileno del 1 bajo visión directa, y se procede a cierre del colgajo dermograso, retirando por contra apertura drenajes a nivel de flancos (Figura 4).

La persona tratada evolucionó de forma favorable, al segundo día de posoperatorio se realiza estudio radiológico contrastado, en el cual se observa buen paso del material de contraste, sin fugas, por lo que se inicia vía oral, retirando los drenajes de cavidad a las 72 horas, los de la abdominoplastia permanecieron siete días. La estancia intrahospitalaria fue de cinco días.

\section{DISCUSIÓN}

La UM posterior a DGY se reporta en $6 \%$ de los sujetos, de los cuales se perforan $1 \%{ }^{4}$ por lo que, en la mayoría de los casos, el tratamiento es el cierre laparoscópico primario. ${ }^{4}$ Estos casos típicamente sufren de dolor abdo- minal persistente, así como anemia secundaria a sangrado crónico de la úlcera e intolerancia a la vía oral debido a la inflamación y estenosis del sitio de la lesión. ${ }^{5}$ En este caso, la paciente presenta datos de abdomen agudo posterior a intervención quirúrgica de abdominoplastia, lo que ameritó una cirugía de urgencia; cabe mencionar que cuenta con factores predisponentes como estrés posquirúrgico y el uso de AINE a dosis altas.

A pesar de factores de riesgo reconocidos como el tabaquismo, estrés, uso de AINE y esteroides, hasta 20\% de los pacientes con úlcera marginal perforada (UMP) no cuentan con estos antecedentes. ${ }^{6}$

Dado que los procedimientos quirúrgicos para la obesidad se han incrementado, cada vez existen más casos con la necesidad de cirugía de contorno corporal; al reportar que el $42 \%$ de ellos se realiza alguna intervención dentro de los primeros cinco años, la abdominoplastia representa $61.7 \%$ del total de los procedimientos reconstructivos.

El antecedente quirúrgico de DGY, cuadro clínico y tomografía con contraste oral son los principales elementos para realizar el diagnóstico de UMP. La tomografía es el estudio de imagen con más sensibilidad, el cual tiene como hallazgos principales aire libre en cavidad abdominal, fuga de material de contraste de la anastomosis gastroyeyunal y líquido libre. Dichos elementos son los que nos llevaron al diagnóstico en esta investigación.

Reoperar a un paciente en el posquirúrgico inmediato de una cirugía estética, como es la abdominoplastia con plicatura de músculos rectos abdominales, implica un gran reto para el grupo quirúrgico. En caso de que se decida abordaje abierto, se altera la estética, la cual fue el motivo de su cirugía, así como aumento en los días de estancia intrahospitalaria y mayor índice de complicaciones. El abordaje laparoscópico convencional es difícil de realizar, ya que la abdominoplastia y la plicatura de rectos impiden

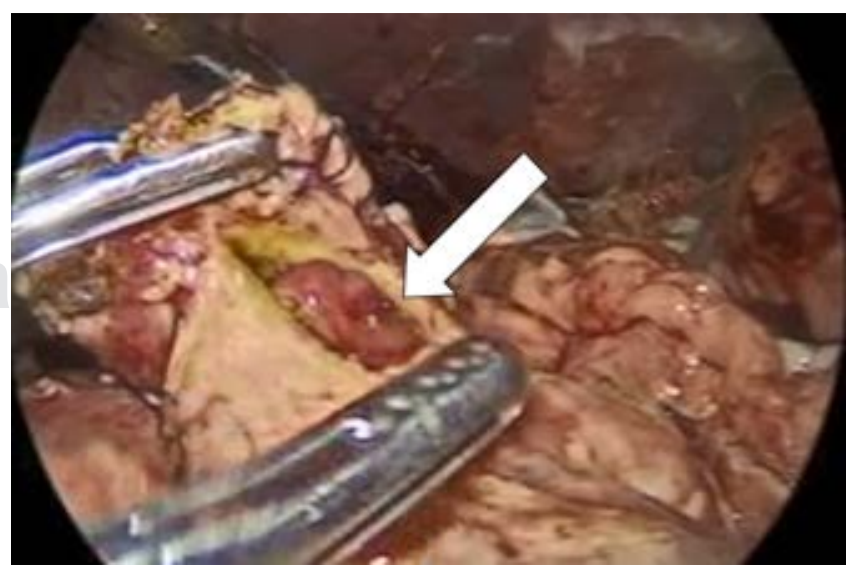

Figura 3: Perforación a nivel de la anastomosis. 


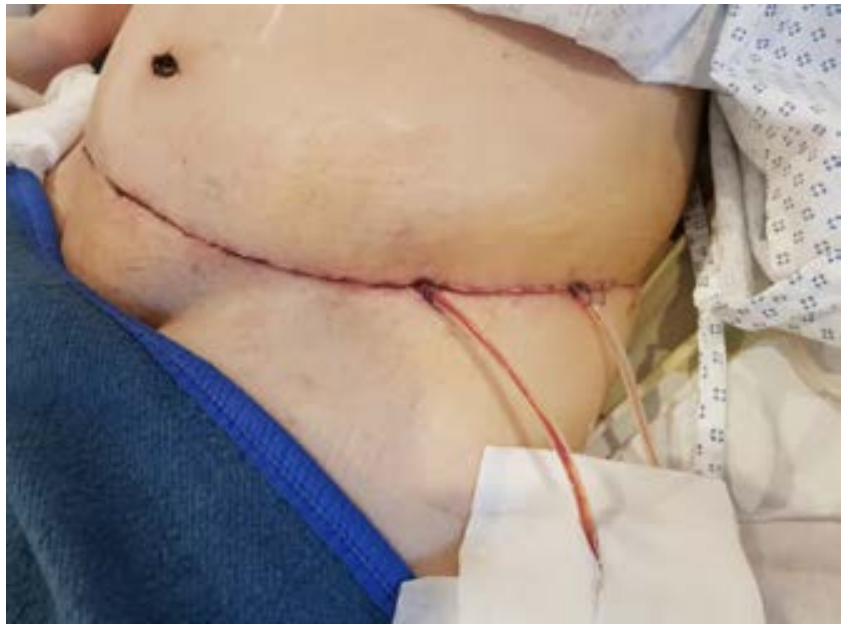

Figura 4: Afrontamiento de colgajo dermograso y colocación de drenajes.

un neumoperitoneo adecuado. En este caso, se decide un abordaje laparoscópico no convencional realizando desmantelamiento de colgajo dermograso y retiro de plicatura de rectos, con lo cual se obtiene un neumoperitoneo ideal y una visión adecuada. Cabe aclarar que este abordaje quirúrgico es el primer reporte en la literatura.

\section{CONCLUSIONES}

La identificación de los factores de riesgo para úlcera marginal perforada en un paciente con derivación gastroyeyunal es importante para el diagnóstico oportuno y su manejo adecuado. Si la úlcera marginal perforada se presenta en el posquirúrgico inmediato de una abdominoplastia, reco- mendamos el desmantelamiento del colgajo dermograso y retiro de plicatura de rectos para realizar el abordaje laparoscópico directamente sobre la aponeurosis, con la finalidad de obtener una adecuada visión de los órganos abdominales, movilización fácil de los instrumentos por el cirujano y conservar el motivo estético de la cirugía primaria.

\section{REFERENCIAS}

1. Spaniolas K, Yang J, Crowley S, Yin D, Docimo S, Bates AT et al. Association of long-term anastomotic ulceration after Roux-en-Y gastric bypass with tobacco smoking. JAMA Surg. 2018; 153: 862-864.

2. Pyke O, Yang J, Cohn T, Yin D, Docimo S, Talamini MA et al. Marginal ulcer continues to be a major source of morbidity over time following gastric bypass. Surg Endosc. 2019; 33: 3451-3456.

3. Altieri MS, Pryor A, Yang J, Yin D, Docimo S, Bates A et al. The natural history of perforated marginal ulcers after gastric bypass surgery. Surg Endosc. 2018; 32: 1215-1222.

4. Wang E, Blackham R, Tan J, Hamdorf J. Giant perforated marginal ulcer after laparoscopic Roux-en-Y gastric bypass. BMJ Case Rep. 2017; 2017: bcr2016218829.

5. Chau E, Youn H, Ren-Fielding CJ, Fielding GA, Schwack BF, Kurian MS. Surgical management and outcomes of patients with marginal ulcer after Roux-en-Y gastric bypass. Surg Obes Relat Dis. 2015; 11: 1071-1075.

6. Moon RC, Teixeira AF, Goldbach M, Jawad MA. Management and treatment outcomes of marginal ulcers after Roux-en-Y gastric bypass at a single high volume bariatric center. Surg Obes Relat Dis. 2014; 10: 229-234.

7. Lazzati A, Katsahian S, Maladry D, Gerard E, Gaucher S. Plastic surgery in bariatric patients: a nationwide study of 17,000 patients on the national administrative database. Surg Obes Relat Dis. 2018; 14: 646-651. 